International Journal of Bifurcation and Chaos, Vol. 11, No. 8 (2001) 2123-2131

(c) World Scientific Publishing Company

\title{
CODIMENSION TWO BIFURCATIONS IN A PREDATOR-PREY SYSTEM WITH GROUP DEFENSE
}

\author{
DONGMEI XIAO* \\ Department of Mathematics, Central China Normal University, \\ Wuhan, Hubei 430079, China \\ SHIGUI RUAN ${ }^{\dagger}$ \\ Department of Mathematics and Statistics, Dalhousie University, \\ Halifax, Nova Scotia, Canada B3H 3J5
}

Received June 1, 2000; Revised November 8, 2000

\begin{abstract}
In this paper we study the qualitative behavior of a predator-prey system with nonmonotonic functional response. The system undergoes a series of bifurcations including the saddle-node bifurcation, the supercritical Hopf bifurcation, and the homoclinic bifurcation. For different parameter values the system could have a limit cycle or a homoclinic loop, or exhibit the socalled "paradox of enrichment" phenomenon. In the generic case, the model has the bifurcation of cusp-type codimension two (i.e. the Bogdanov-Takens bifurcation) but no bifurcations of codimension three.
\end{abstract}

\section{Introduction}

In a well-known paper, Rosenzweig [1971] considered exploitation in a two-species ecosystem with a vertical isocline (no intraspecific interaction) for the predator and with prey limited by their environment to a finite carrying capacity that was an increasing function of their food supply. By performing a linear stability analysis of the community equilibrium point of the governing system of differential equations for a series of models, Rosenzweig showed the destabilization of a stable predatorprey system could be accomplished by enriching the predators' food supply sufficiently to result in the extinction of both species. This phenomenon is the so-called paradox of enrichment. Though Rosenzweig's predictions are controversial, there is experimental evidence that supports the warning (see e.g. [Luckinbill, 1973; Schaffer \& Rosenzweig, 1978], etc.). Recently, some researchers have provided support to Rosenzweig's predictions by mathematically analyzing certain predator-prey models, for instance, the predator-prey models with group defense.

In population dynamics, group defense is a term used to describe the phenomenon whereby predation is decreased, or even prevented altogether, due to the increased ability of the prey to better defend or disguise themselves when their numbers are large enough. An example of this phenomenon is described by Tener [1965]. Lone musk ox can be successfully attacked by wolves. Small herds of musk ox (2-6 animals) are attacked but with rare success. No successful attacks have been observed in larger herds. A second example described by Holmes and Bethel [1972] involves certain

\footnotetext{
${ }^{*}$ Research supported by the National Natural Science Foundations of China, No. 10071027.

E-mail: dmxiao@ccnu-1.ccnu.edu.cn

${ }^{\dagger}$ Research supported by the Natural Science and Engineering Research Council of Canada.

E-mail: ruan@mathstat.dal.ca
} 
insect populations. Apparently, large swarms of the insects make individual identification difficult for their predators. The third example was observed by Davidowicz et al. [1988]. Filamenteous algae are often qualified as inedible by herbivorous zooplankton. However, experiments show that Daphnia can consume them at low concentrations, and jam their filtering apparatus in high concentrations.

To study the predator-prey interaction when the prey exhibits group defense, Freedman and Wolkowicz [1986], Mischaikow and Wolkowicz [1990] and Wolkowicz [1988] proposed and studied the following model

$$
\begin{aligned}
& \dot{x}=x g(x, K)-y p(x), \\
& \dot{y}=y(-D+q(x)) .
\end{aligned}
$$

Here, $x$ and $y$ are functions of time representing population densities of prey and predator, respectively; $K>0$ is the carrying capacity of the prey and $D>0$ is the death rate of the predator. The function $g(x, K)$ represents the specific growth rate of the prey in the absence of predator and is assumed to satisfy the following conditions (for $x \geq 0$, $K>0)$

$$
\begin{gathered}
g(K, K)=0, \quad g(0, K)>0, \lim _{K \rightarrow \infty} g(0, K)<\infty \\
g_{x}(x, K)<0, g_{K}(x, K) \geq 0, g_{x K}(x, K)>0 \\
\lim _{K \rightarrow \infty} g_{x}(x, K)=0 .
\end{gathered}
$$

A prototype is the logistic growth

$$
g(x, K)=r\left(1-\frac{x}{K}\right)
$$

which satisfies all the conditions. The function $p(x)$ denotes the predator response function and it is assumed that $p(x)$ satisfies

$$
p(0)=0, \quad p(x)>0 \quad \text { for } x>0 .
$$

In order to model group defense, it is assumed that there is a constant $M>0$ such that (see Fig. 1)

$$
p^{\prime}(x) \begin{cases}>0, & 0 \leq x<M \\ <0, & x>M .\end{cases}
$$

In particular, the function $p(x)=\alpha x e^{-\beta x}$ proposed in [Freedman \& Ruan, 1992] satisfies the assumptions, where $\alpha$ and $\beta$ are positive constants. The rate of conversion of prey to predator is described by $q(x)$ which has properties similar to $p(x)$. So

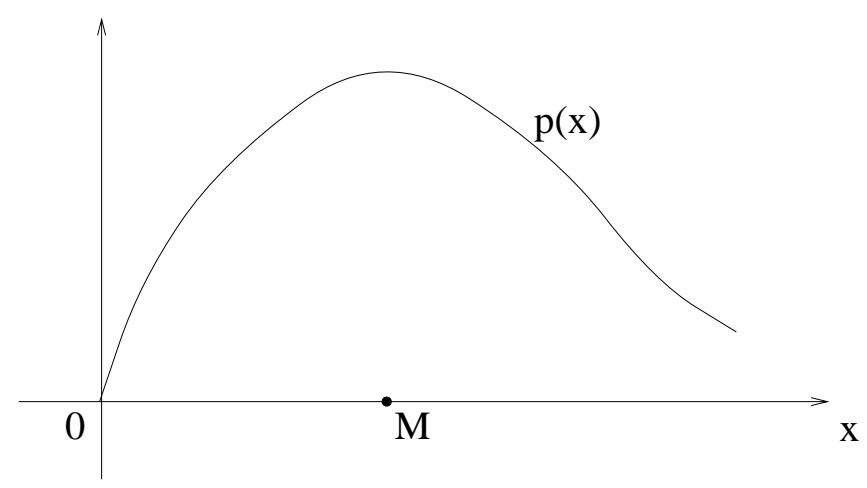

Fig. 1. The nonmonotonic predator response function $p(x)$.

usually it is assumed that $q(x)=\mu p(x)$ for some positive constant $\mu$.

Freedman and Wolkowicz [1986] showed that if the carrying capacity of the prey population is sufficiently large, the predator population is almost always driven to extinction. This supports the phenomenon of "paradox of enrichment." In the case when there are two interior equilibria, by using the carrying capacity of the prey $K$ as a bifurcation parameter, Wolkowicz [1988] showed, among other things, that system (1) undergoes a Hopf bifurcation at the inside equilibrium when $K$ passes a critical value and there is a homoclinic bifurcation associated with the Hopf bifurcation (either the Hopf bifurcation followed by the homoclinic bifurcation or the homoclinic bifurcation followed by the Hopf bifurcation) when the same bifurcation parameter $K$ takes some other value. The observation and analysis made by Freedman and Wolkowicz [1986] and Wolkowicz [1988] indicate that the codimension of these bifurcations in system (1) is greater than or equal to two.

The purpose of this paper is to discuss the dynamics of a predator-prey system of the form

$$
\begin{aligned}
& \dot{x}=r x\left(1-\frac{x}{K}\right)-\alpha x y e^{-\beta x}, \\
& \dot{y}=y\left(\mu \alpha x e^{-\beta x}-D\right),
\end{aligned}
$$

where $x$ and $y$ represent the prey and predator populations, respectively; $r, K, \alpha, \beta, \mu$ and $D$ are positive parameters. For the sake of simplicity, we scale the parameter $\alpha$ by letting $\bar{y}=\alpha y$ and $\bar{\mu}=\mu \alpha$. Dropping the bars, we have the following system

$$
\begin{aligned}
& \dot{x}=r x\left(1-\frac{x}{K}\right)-x y e^{-\beta x}, \\
& \dot{y}=y\left(\mu x e^{-\beta x}-D\right),
\end{aligned}
$$


From the point of view of biology, we only restrict our attention to system (3) in the closed first quadrant in the $(x, y)$ plane. We shall make global qualitative analysis and bifurcation analysis of system (3) depending on all parameters and show that system (3) has a unique limit cycle or a global attractive equilibrium in the interior of the first quadrant, or exhibits the "paradox of enrichment" phenomenon for different parameter values. Furthermore, we show that system (3) can exhibit qualitatively different dynamics including the supercritical Hopf bifurcation, the saddle-node bifurcation as well as the homoclinic bifurcation. In the generic case, system (3) has the bifurcation of codimension two (i.e. the Bogdanov-Takens bifurcation) and no bifurcations of codimension three.

We would like to point out that though the response function $p(x)=\alpha x e^{-\beta x}$ looks similar to the Ivlev's response function $p(x)=\alpha x\left(1-e^{-\beta x}\right)$, we will see that system (3) exhibits much richer and more complicated dynamics than the predator-prey model with Ivlev's functional response (see [Sugie, 1998; Kooij \& Zegeling, 1996]). The key difference is that system (3) has a Bogdanov-Takens singularity while the the predator-prey model with Ivlev's functional response does not. The difference has also been observed between a predatorprey model with the simplified Monod-Haldane response function $p(x)=m x /\left(a+x^{2}\right)$ [Ruan \& Xiao, 2001] and predator-prey systems with Holling typeII response function $p(x)=m x /(a+x)$ and Holling type-III response function $p(x)=m x^{2} /\left(a+x^{2}\right)$ (see cf. [Kooij \& Zegeling, 1997; Sugie et al., 1997] and the references cited therein).

This paper is organized as follows. Existence of equilibria and nonexistence of closed orbits of the system (3) are discussed in Sec. 2. In Sec. 3 we study all possible bifurcations depending on all parameters. Using $K$ (the carrying capacity of the prey) and $D$ (the death rate of the predator) as bifurcation parameters, we obtain the versal unfolding of the Bogdanov-Takens singularity. For some parameter values, the system has a unique limit cycle or a homoclinic loop. A brief discussion is given in Sec. 4.

\section{General Phase Portraits Analysis of the System (3)}

As it is typical for predator-prey systems, the $x$ axis, $y$-axis and the interior of the first quadrant are all invariant under system (3), solutions with positive initial values are positive and bounded, and there are a hyperbolic saddle point at the origin and an equilibrium $(K, 0)$ in the $x$-axis for all permissible parameters. The equilibria of the greatest interest are those interior to the first quadrant, so we seek conditions for such equilibria to exist. From system (3), we see an interior equilibrium, the following equation

$$
\mu x e^{-\beta x}-D=0
$$

must have a positive root $x^{*}$. Therefore, the first condition is that

$$
\mu \geq e \beta D
$$

To determine the $y$ value of the equilibrium, we merely solve

$$
r x\left(1-\frac{x}{K}\right)-x y e^{-\beta x}=0
$$

for $y$ at the root $x^{*}$ and get

$$
y^{*}=r\left(1-\frac{x^{*}}{K}\right) e^{\beta x^{*}}
$$

To ensure that $y^{*}>0$, however, we must make a second assumption

$$
x^{*}<K \text {. }
$$

Notice that if the predator response function is monotone, Freedman [1987] has shown that if $x^{*}>K$, then the predator goes extinct. If predator

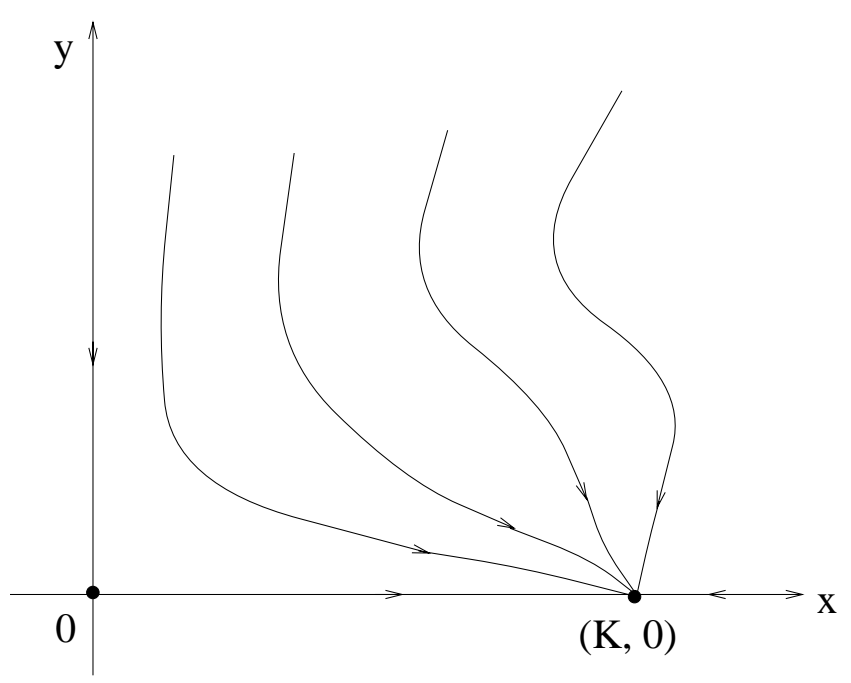

Fig. 2. The phase portrait of system (3) when $\mu<e \beta D$ or $\mu=e \beta D$ and $\beta K \leq 1$. 
response function is nonmonotone as in system (3), there could be two equilibria since $r x(1-(x / K))-$ $x y e^{-\beta x}=0$ may have two roots $x_{1}$ and $x_{2}$. Even if one $x_{i}>K$, the predator does not necessarily go to extinction. We will see more details in the following.

Note that if condition (4) is not satisfied, then system (3) has no interior equilibria; if (4) holds, then system (3) may have one or two interior equilibria. Condition (5) is required when interior equilibria exist. Next we discuss the all possible phase portraits of system (3) depending on all parameters.

\subsection{Either $\mu<e \beta D$ or $\mu=e \beta D$ and $\beta K \leq 1$}

In this case, system (3) has no interior equilibria. It is easy to see that the equilibrium $(K, 0)$ is a stable node. The phase portrait is depicted in Fig. 2.

\section{2. $\mu=e \beta D$ and $\beta K>1$}

In this case, system (3) has three equilibria, a hyperbolic saddle $(0,0)$, a stable node $(K, 0)$ and an interior equilibrium $\left(x_{0}, y_{0}\right)$, where $x_{0}=(1 / \beta)$, $y_{0}=r(1-(1 / \beta K)) e$.

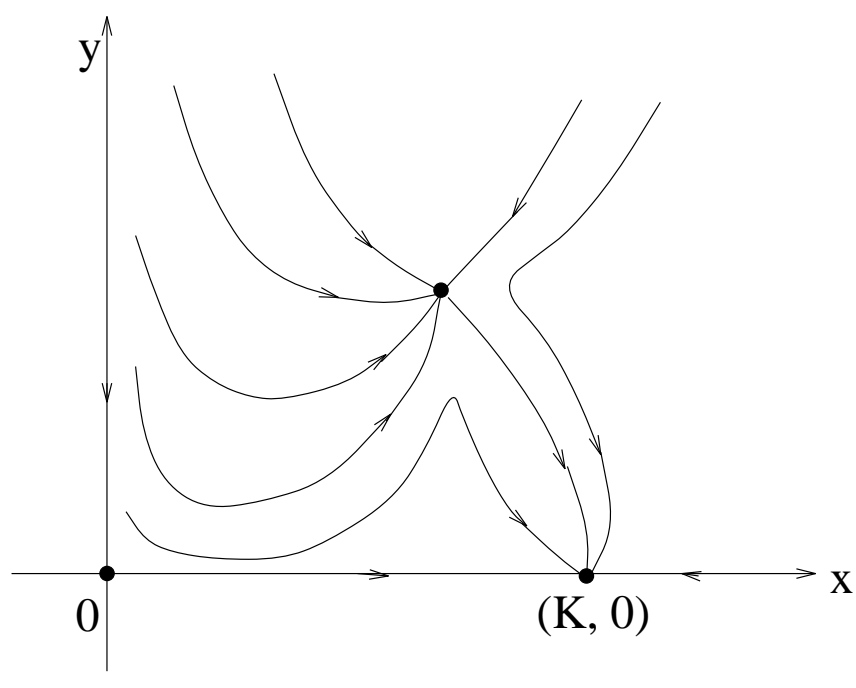

(a)

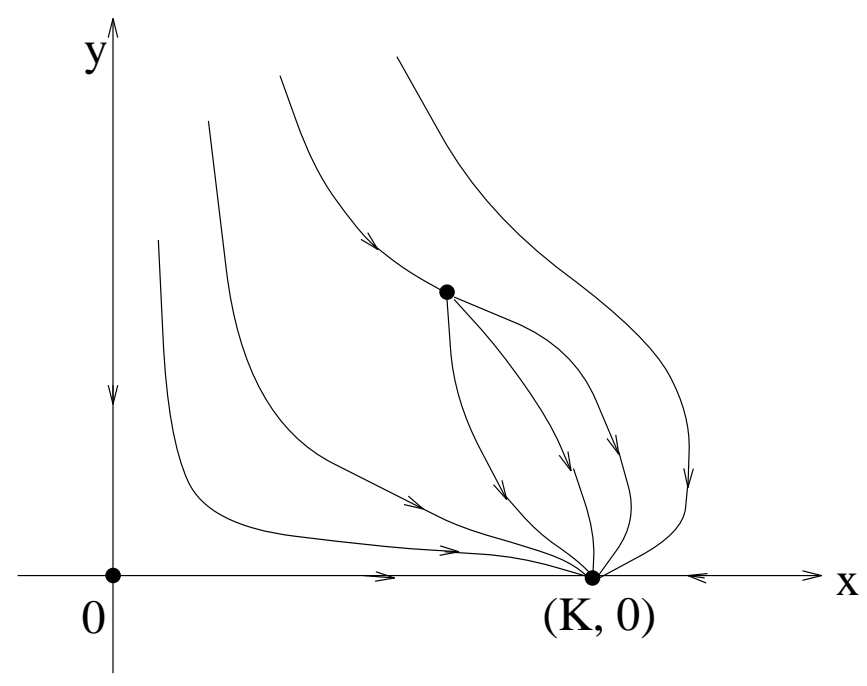

(b)

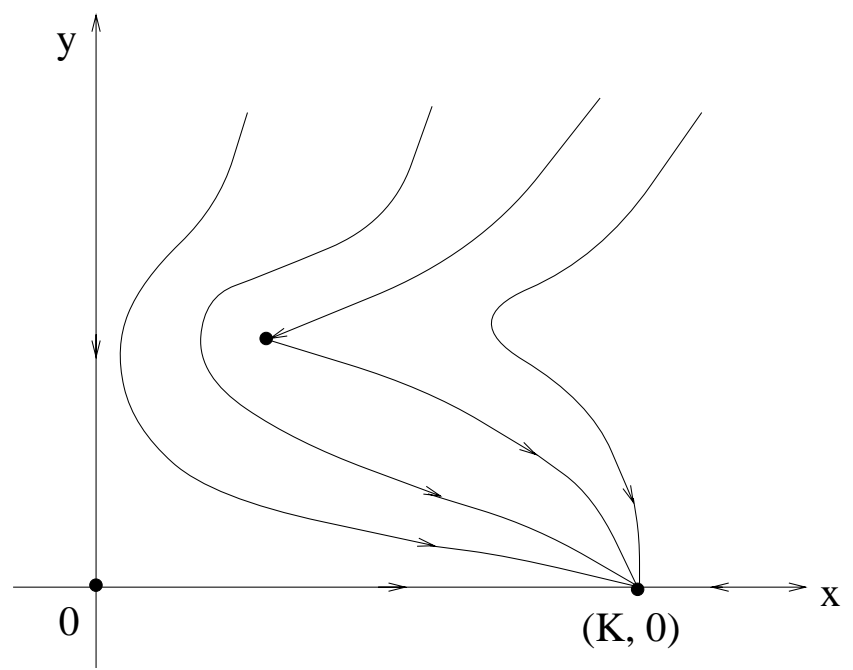

(c)

Fig. 3. The phase portrait of system (3) when (a) $\mu=e \beta D$ but $(1 / \beta)<K<(2 / \beta)$; (b) $\mu=e \beta D$ but $K>(2 / \beta)$; (c) $\mu=e \beta D$ and $K=1 / \beta$. 
After some calculations, we know that $\left(x_{0}, y_{0}\right)$ is a saddle-node when $K \neq(2 / \beta)$ and a cusp when $K=2 / \beta$. In both cases system (3) has no closed orbits. Detailed information can be seen in Fig. 3 . Especially, from Figs. 3(b) and 3(c) we can easily see that sufficient enrichment of the environment leads to the extinction of the predator for almost all initial values, which strongly supports the so-called "paradox of enrichment".

\section{3. $\mu>e \beta D$}

In this case, system (3) has at most four equilibria, $(0,0),(K, 0)$, and two interior equilibria $\left(x_{1}, y_{1}\right)$, $\left(x_{2}, y_{2}\right)$, where $x_{1}$ and $x_{2}$ are roots of

$$
\mu x e^{-\beta x}-D=0 \text {. }
$$

Obviously, $0<x_{1}<(1 / \beta)<x_{2}$.

More precisely, there are three possibilities. (i) When $K<x_{1}$, system (3) has two boundary equilibria, $(0,0)$ and $(K, 0)$, and no interior equilibria. The dynamics of system (3) is trivial as in Fig. 2. (ii) When $x_{1}<K<x_{2}$, system (3) has three equilibria: two hyperbolic saddles $(0,0)$ and $(K, 0)$, and an interior equilibrium $\left(x_{1}, y_{1}\right)$. In this case the interior equilibrium $\left(x_{1}, y_{1}\right)$ is a focus or node, which is stable (unstable) if $x_{1}<K<x_{1}+\beta^{-1} \leq$ $x_{2}\left(x_{1}+\beta^{-1}<K<x_{2}\right.$, respectively). (iii) When $x_{2}<K$, system (3) has four equilibria: a hyperbolic saddle $(0,0)$, a hyperbolic stable node $(K, 0)$ and two interior equilibria $\left(x_{1}, y_{1}\right)$ and $\left(x_{2}, y_{2}\right)$. We can see that $\left(x_{2}, y_{2}\right)$ is a hyperbolic saddle and $\left(x_{1}, y_{1}\right)$ is a focus or node, which is stable (unstable) if $x_{2}<K<x_{1}+\beta^{-1}\left(x_{2} \leq x_{1}+\beta^{-1}<\right.$ $K$, respectively).

In case 2.3 (ii) and (iii), system (3) could have complicated dynamics in the interior of the first quadrant. Next we give two theorems on nonexistence of closed orbits of system (3).

Theorem 2.1. If $e \beta D<\mu$ and $x_{1}<K \leq 1 / \beta$, then system (3) has three equilibria: two hyperbolic saddles $(0,0)$ and $(K, 0)$, and a globally asymptotically stable equilibrium $\left(x_{1}, y_{1}\right)$ in the interior of the first quadrant. The phase portrait is shown in Fig. 4.

Proof. It can be easily checked that the equilibrium $\left(x_{1}, y_{1}\right)$ is a stable focus (or node). Next we prove that system (3) has no periodic orbits in the interior of the first quadrant.

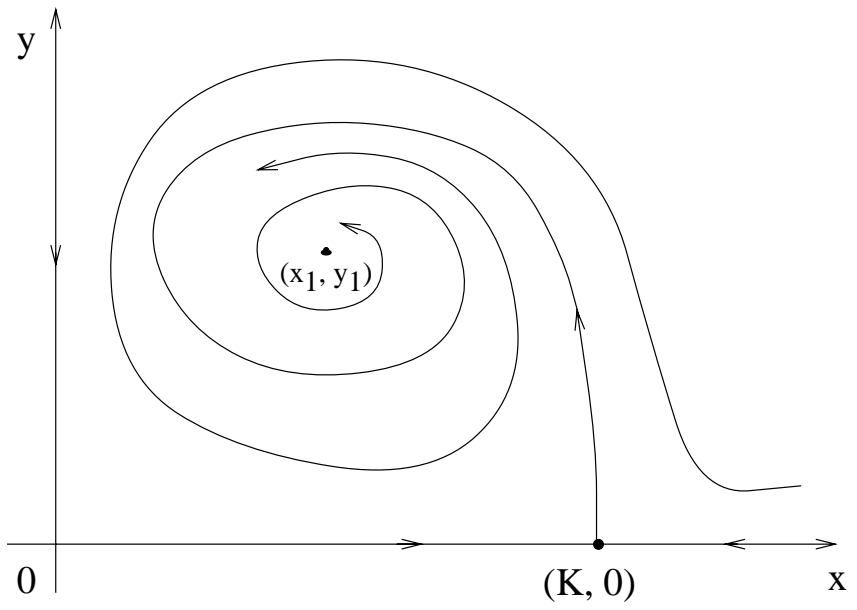

Fig. 4. The phase portrait of system (3) when $e \beta D<\mu$ and $x_{1}<K \leq(1 / \beta)$.

Introduce the new time $\tau$ by $d t=e^{\beta x} d \tau$, system (3) is equivalent to

$$
\begin{aligned}
& \frac{d x}{d \tau}=r x\left(1-\frac{x}{K}\right) e^{\beta x}-x y=f(x, y) \\
& \frac{d y}{d \tau}=\mu x y-D y e^{\beta x}=g(x, y) .
\end{aligned}
$$

Taking the Dulac function $D(x, y)=x^{-1} y^{-1}$ for system (6), we have

$$
\begin{gathered}
\frac{\partial[D(x, y) f(x, y)]}{\partial x}+\frac{\partial[D(x, y) g(x, y)]}{\partial y} \\
=r y^{-1} e^{\beta x}\left(\beta-\frac{1}{K}-\frac{\beta x}{K}\right)<0
\end{gathered}
$$

as $K \leq(1 / \beta)$ in the interior of the first quadrant. Thus, system (6) has no closed orbits in the interior of the first quadrant by Dulac-Bendixson criterion. Hence, the equilibrium $\left(x_{1}, y_{1}\right)$ is globally asymptotically stable in the interior of the first quadrant.

Theorem 2.2. If $\mu>e \beta D$ and $K>(1 / \beta)+x_{2}$, then system (3) has four equilibria: two hyperbolic saddles $(0,0)$ and $\left(x_{2}, y_{2}\right)$, a hyperbolic stable node $(K, 0)$ and an unstable equilibrium $\left(x_{1}, y_{1}\right)$. Moreover, system (3) has no closed orbits, that is, it exhibits the so-called "paradox of enrichment" phenomenon. The phase portrait is given in Fig. 5.

Proof. Clearly the equilibrium $\left(x_{1}, y_{1}\right)$ is an unstable focus as $K>(1 / \beta)+x_{2}$. Since the equilibrium $\left(x_{2}, y_{2}\right)$ is a saddle, a closed orbit (a periodic orbit 


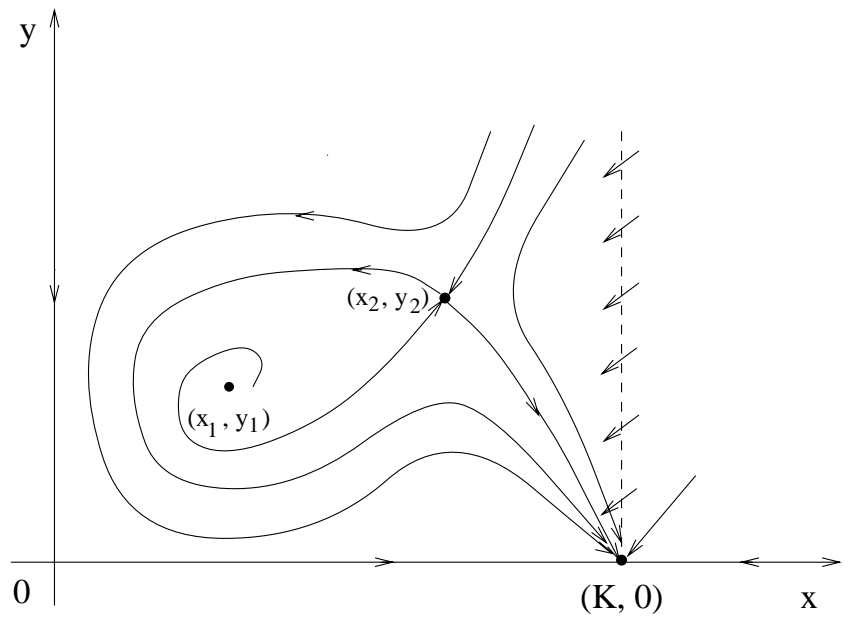

Fig. 5. The phase portrait of system (3) when $\mu>e \beta D$ and $K>(1 / \beta)+x_{2}$.

or a homoclinic loop) of system (3) must be in the domain $E$ if it exists, here

$$
E=\left\{(x, y): 0<x \leq x_{2}, 0<y<+\infty\right\} .
$$

Note that the problem of existence of a closed orbit of system (3) in $E$ is equivalent to that of system (6) in $E$. As in the proof of Theorem 2.1, we have

$$
\begin{gathered}
\frac{\partial[D(x, y) f(x, y)]}{\partial x}+\frac{\partial[D(x, y) g(x, y)]}{\partial y} \\
=r K^{-1} y^{-1} e^{\beta x}(K \beta-1-\beta x) \\
>r \beta K^{-1} y^{-1} e^{\beta x}\left(x_{2}-x\right) \geq 0
\end{gathered}
$$

as $K>(1 / \beta)+x_{2}$ in $E$. Therefore, system (3) has neither a limit cycle nor a homoclinic loop in the interior of the first quadrant. This completes the proof.

Remark 2.3. Notice that for the original system (2), the condition (4) becomes

$$
\mu \alpha \geq e \beta D
$$

Accordingly, we can obtain all the phase portraits of the system (2).

\section{Multiple Bifurcations of System (3)}

According to the analysis in Sec. 2, we know that there exist some special parameter values such that the equilibrium of system (3) is not hyperbolic.
Thus, when the parameters vary in the neighborhood of these special values, the dynamics of system (3) can change, which leads to bifurcations. In this section, we discuss all possible bifurcations of system (3) at these equilibria, and show that system (3) has a unique limit cycle or a homoclinic loop for different parameter values.

First of all, we need the model to be persistent from the point of view of biology, hence, the condition $\mu \geq e \beta D$ is required. On the other hand, mathematically the following surface

$$
S N=\left\{(\mu, \beta, D, K): \mu=e \beta D \text { and } K \neq \frac{2}{\beta}\right\}
$$

is a saddle-node bifurcation surface. When the parameters pass from one side of this surface to the other side, the number of equilibria of system (3) changes, i.e. from zero to two. Thus, system (3) exhibits saddle-node bifurcation.

However, when $\mu=e \beta D$ and $K=2 / \beta$, system (3) has an interior equilibrium $\left(x_{0}, y_{0}\right)$ which is a cusp from Sec. 2. Moreover, we have

Theorem 3.1. If $\mu=e \beta D$ and $K=2 / \beta$, then the equilibrium $\left(x_{0}, y_{0}\right)$ of system (3) is a cusp of codimension two, i.e. it is a Bogdanov-Takens singularity.

Proof. When $\mu=e \beta D$ and $K=2 / \beta$, we have $x_{0}=1 / \beta, y_{0}=r e / 2$. Let $X=x-x_{0}, Y=y-y_{0}$, we can rewrite system (3) as

$$
\begin{aligned}
& \dot{X}=-\frac{1}{\beta e} Y-\frac{r \beta}{4} X^{2}+P(X, Y), \\
& \dot{Y}=-\frac{r \beta \mu}{4} X^{2}+Q(X, Y),
\end{aligned}
$$

where $P(X, Y)=O\left(|(X, Y)|^{3}\right)$ and $Q(X, Y)=$ $O\left(|(X, Y)|^{3}\right)$ are $C^{\infty}$ functions in $(X, Y)$. In the small neighborhood of the origin, we transform system (8) into a simpler form

$$
\begin{aligned}
& \dot{x}=y, \\
& \dot{y}=\frac{r \mu}{4 e} x^{2}-\frac{r \beta}{2} x y+R(x, y)
\end{aligned}
$$

by smooth invertible transformations

$$
x=X, \quad y=-\frac{1}{\beta e} Y-\frac{r \beta}{4} X^{2}+P(X, Y),
$$

where $R(x, y)=O\left(|(x, y)|^{3}\right)$ is a $C^{\infty}$ function of their arguments. 
Since the coefficients of the terms $x^{2}$ and $x y$ in system (9) are not zero, the origin of system (9) is a cusp of codimension two, i.e. a Bogdanov-Takens singularity. This completes the proof.

For a Bogdanov-Takens singularity, it is more interesting to find the versal unfolding depending on the original parameters in system (3). For this, we choose the carrying capacity of the prey population $K$ and the death rate of predator $D$ in system (3) as bifurcation parameters, and consider

$$
\begin{aligned}
& \dot{x}=r x\left[1-\left(\lambda_{1}+\frac{1}{K_{0}}\right) x\right]-x y e^{-\beta_{0} x}, \\
& \dot{y}=y\left(\mu_{0} x e^{-\beta_{0} x}-\lambda_{2}-D_{0}\right)
\end{aligned}
$$

where $\lambda_{1}$ and $\lambda_{2}$ are parameters which vary in a small neighborhood of the origin; $\mu_{0}, K_{0}, \beta_{0}$ and $D_{0}$ satisfy the equations $\mu=e \beta D$ and $K=2 / \beta$. We translate the origin of system (10) to the point $\left(x_{0}, y_{0}\right)=(1 / \beta, r e / 2)$, then system (10) becomes

$$
\begin{aligned}
\dot{X}= & -r \beta_{0}^{-2} \lambda_{1}-2 r \beta_{0}^{-1} \lambda_{1} X-\frac{1}{\beta_{0} e} Y \\
& -\left(\frac{r \beta_{0}}{4}+2 r \lambda_{1}\right) X^{2}+P(X, Y), \\
\dot{Y}= & -\frac{r e}{2} \lambda_{2}-\lambda_{2} Y-\frac{r \beta_{0} \mu_{0}}{4} X^{2}+Q(X, Y) .
\end{aligned}
$$

Following the procedure of deriving the normal form in [Xiao \& Ruan, 1999], we take $x_{1}=X$, $x_{2}=-r \beta_{0}^{-2} \lambda_{1}-2 r \beta_{0}^{-1} \lambda_{1} X-\left(1 / \beta_{0} e\right) Y-\left(\left(r \beta_{0} / 4\right)+\right.$ $\left.2 r \lambda_{1}\right) X^{2}+P(X, Y)$, then system (11) is written as

$$
\begin{aligned}
\dot{x}_{1}= & x_{2}, \\
\dot{x}_{2}= & \frac{r}{2 \beta_{0}} \lambda_{2}-\left(\frac{2 r}{\beta_{0}} \lambda_{1}+\lambda_{2}\right) x_{2}+\frac{r \mu_{0}}{4 e} x_{1}^{2} \\
& -\frac{r \beta_{0}}{2} x_{1} x_{2}+\bar{R}(x, \lambda),
\end{aligned}
$$

here $\bar{R}(x, \lambda)=O\left(|\lambda|^{2}\right)+O\left(|\lambda|^{2} x\right)+O((|\lambda|+$ $\left.|x|)|x|^{2}\right)$ is a smooth function of its arguments. We simply denote system (12) by

$$
\begin{aligned}
& \dot{x}_{1}=x_{2}, \\
& \dot{x}_{2}=\tau_{1}+\tau_{2} x_{2}+\frac{r \mu_{0}}{4 e} x_{1}^{2}-\frac{r \beta_{0}}{2} x_{1} x_{2}+\bar{R}(x, \tau),
\end{aligned}
$$

where $\tau_{1}=\left(r / 2 \beta_{0}\right) \lambda_{2}$ and $\tau_{2}=-\left(\left(2 r / \beta_{0}\right) \lambda_{1}+\right.$ $\left.\lambda_{2}\right)$, which vary in a small neighborhood of the origin.

By the theorems in [Bogdanov, 1981a, 1981b], we obtain the following local representations of the bifurcation curves in a small neighborhood of the origin:

1. The saddle-node bifurcation curve $S N=$ $\left\{\left(\lambda_{1}, \lambda_{2}\right): \lambda_{2}=0, \lambda_{1} \neq 0\right\}$.

2. The Hopf bifurcation curve $H=\left\{\left(\lambda_{1}, \lambda_{2}\right)\right.$ : $\left.\lambda_{2}<0, r \sqrt{-\left(\beta_{0} e / 2 \mu_{0}\right) \lambda_{2}}=2 r \beta_{0}^{-1} \lambda_{1}+\lambda_{2}\right\}$.

3 . The homoclinic bifurcation curve $H L=$ $\left\{\left(\lambda_{1}, \lambda_{2}\right): \lambda_{2}<0,(5 r / 7) \sqrt{-\left(\beta_{0} e / 2 \mu_{0}\right) \lambda_{2}}=\right.$ $\left.2 r \beta_{0}^{-1} \lambda_{1}+\lambda_{2}\right\}$.

We sketch these bifurcation curves in a small neighborhood of the origin in the $\left(\lambda_{1}, \lambda_{2}\right)$ plane by their first approximations and obtain the following bifurcation diagram (see Fig. 6). The bifurcation curves divide the parameter plane into four parts: I, II, III and IV.

(a) When the parameter values are in the region III, system (10) has two interior equilibria and a unique stable limit cycle. Thus there exists an open set of initial population densities such that both the predator and the prey tend to a stable oscillation.

(b) When the parameter values lie on the homoclinic bifurcation curve $H L$, system (10) has two interior equilibria, a stable homoclinic loop and no limit cycle.

Remark 3.2. By Theorem 3.1 and the above argument, we know that system (3) cannot undergo bifurcations of codimension three at the degenerate singularity.

In the case 2.3 of Sec. 2 , we can see that the stability of the equilibrium $\left(x_{1}, y_{1}\right)$ can change as the parameter $K$ varies, which implies that the equilibrium $\left(x_{1}, y_{1}\right)$ is not hyperbolic. Next we discuss the property of $\left(x_{1}, y_{1}\right)$.

Lemma 3.3. If $\mu>e \beta D$ and $K=x_{1}+\beta^{-1}$, then the equilibrium $\left(x_{1}, y_{1}\right)$ of system (3) is a stable multiple focus of multiplicity one.

Proof. Let $\bar{x}=x-x_{1}, \bar{y}=y-y_{1}$. Then when $K=x_{1}+\beta^{-1}$, system (3) can be transferred to

$$
\begin{aligned}
& \dot{\bar{x}}=-x_{1} e^{-\beta x_{1}} \bar{y}+\sum_{i+j \geq 2} \frac{f_{i j}}{i ! j !} \bar{x}^{i} \bar{y}^{j}, \\
& \dot{\bar{y}}=\mu y_{1} e^{-\beta x_{1}}\left(1-\beta x_{1}\right) \bar{x}+\sum_{i+j \geq 2} \frac{g_{i j}}{i ! j !} \bar{x}^{i} \bar{y}^{j},
\end{aligned}
$$




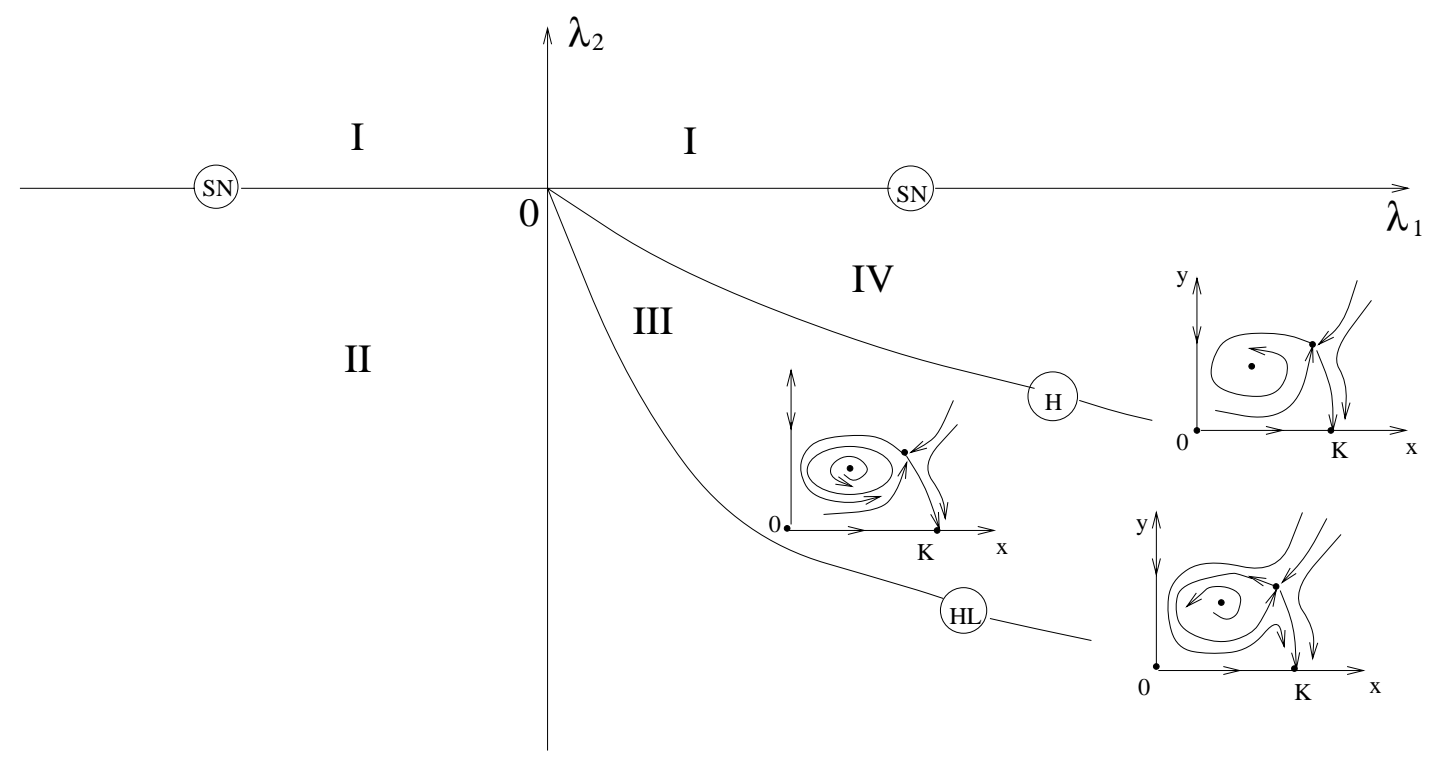

Fig. 6. The bifurcation set and the corresponding phase portraits of system (10).

where $f_{i j}:=\left.\left(\partial^{i+j} f / \partial^{i} x \partial^{j} y\right)\right|_{\left(x_{1}, y_{1}\right)}, g_{i j}:=\left(\partial^{i+j} g /\right.$ $\left.\partial^{i} x \partial^{j} y\right)\left.\right|_{\left(x_{1}, y_{1}\right)}$, and $f:=r x(1-(x / K))-x y e^{-\beta x}$ and $g:=y\left(\mu x e^{-\beta x}-D\right)$.

According to the formula for the third focal value of a multiple focus on p. 253 of [Andronov et al., 1971] (see also [Andronov et al., 1966]), we obtain, after some elementary but lengthy computations, the following expression for the third focal value $\alpha_{3}$ of the multiple focus $(0,0)$ in system (14)

$$
\alpha_{3}=-\frac{3 \pi r \mu \beta x_{1} y_{1}}{4 K e^{2 \beta x_{1}} \theta^{\frac{3}{2}}}\left[2-\left(\beta x_{1}\right)^{2}\right]
$$

where $\theta=\mu x_{1} y_{1}\left(1-\beta x_{1}\right) e^{-2 \beta x_{1}}$. Since $\beta x_{1}<1$, $\alpha_{3}<0$. Hence, the equilibrium $\left(x_{1}, y_{1}\right)$ is a stable multiple focus of multiplicity one. This proves the lemma.

By Lemma 3.3, we know that the equilibrium $\left(x_{1}, y_{1}\right)$ is a stable focus when $\mu>e \beta D$ and $x_{1}+\beta^{-1} \geq K$. However, the equilibrium $\left(x_{1}, y_{1}\right)$ is an unstable focus as $\mu>e \beta D$ and $K>x_{1}+\beta^{-1}$. Therefore, system (3) can undergo a supercritical Hopf bifurcation. A stable limit cycle appears in the small neighborhood of $\left(x_{1}, y_{1}\right)$. The surface

$$
H_{1}=\left\{(K, \mu): K=x_{1}+\beta^{-1}, \mu>e \beta D\right\}
$$

is called the supercritical Hopf bifurcation surface of system (3).

Summarizing the above arguments, we have
Theorem 3.4. There exists a positive number $\varepsilon$ such that system (3) has at least one limit cycle when $\mu>e \beta D$ and $x_{1}+\beta^{-1}+\varepsilon>K>x_{1}+\beta^{-1}$.

According to above analysis and Theorems 2.1 and 2.2 , we make the following conjectures.

Conjecture 3.5. When $\mu>e \beta D$ and $x_{1}+\beta^{-1} \geq$ $K>\beta^{-1}$, system (3) has a unique interior equilibrium $\left(x_{1}, y_{1}\right)$, which is a global attractor in the interior of the first quadrant.

Conjecture 3.6. When $\mu>e \beta D$ and $K>x_{1}+$ $\beta^{-1}$, system (3) has at most one limit cycle, it is stable if it exists.

Remark 3.7. For system (2) with the original parameters, the first condition of Theorem 3.1 becomes $\mu \alpha=e \beta D$. Biologically, Theorem 3.1 indicates that if the death rate of the predator satisfies $D=\mu \alpha / e \beta$ and the carrying capacity of the prey satisfies $K=2 / \beta$, then the unique interior equilibrium of the predator-prey system (2) is a cusp of codimension two, and in the neighborhood of the critical values of $(D, K)$ the system exhibits very rich dynamics. While Theorem 3.4 demonstrates this when the death rate of the predator is decreased so that $D<\mu \alpha / e \beta$ and the carrying capacity $K$ of the prey takes certain value, then the predator and prey populations exist in the form of oscillatory periodic solutions. 


\section{Discussion}

Multiple parameter bifurcation in predator-prey systems is an interesting, important and challenging problem, since it is to determine all possible types of bifurcations depending on all parameters involved in the systems. Rothe and Shafer [1991] investigated a predator-prey system with a nonmonotone functional response of the form

$$
p(x)=\frac{m x}{a+b x+x^{2}}
$$

and showed that it exhibits very rich dynamics such as Hopf, saddle-loop, and semi-stable bifurcations of cycles, as well as a special bifurcation of cycles from infinity. It is also shown that two cycles can coexist. Therefore, their system exhibits bifurcations of codimension three.

In this paper we have considered a predatorprey system with a different nonmonotone functional response, namely,

$$
p(x)=\alpha x e^{-\beta x} .
$$

It has been shown that when the parameters take some critical values, the predator-prey system has a unique interior equilibrium which is a cusp of codimension two and in the small neighborhood of these critical values the system exhibits BogdanovTakens bifurcation. By carrying out a global qualitative analysis of the system depending on all parameters, we have shown that the system has a unique limit cycle or a global attractive equilibrium in the interior of the first quadrant, or exhibits the "paradox of enrichment" phenomenon for different parameter values. Moreover, the system undergoes a series of bifurcations including a supercritical Hopf bifurcation, a saddle-node bifurcation and a homoclinic bifurcation. Generically the system has bifurcations of codimension two and, differing from the system studied by Rothe and Shafer [1991], has no bifurcations of codimension three.

\section{References}

Andronov, A. A., Vitt, E. A. \& Khaiken, S. E. [1966] Theory of Oscillations (Pergamon Press, Oxford).

Andronov, A. A., Leontovich, E. A., Gordon, I. I. \& Maier, A. G. [1971] Theory of Bifurcations of Dynamical Systems on a Plane (Israel Program for Scientific Translations, Jerusalem).

Bogdanov, R. [1981a] "Bifurcations of a limit cycle for a family of vector fields on the plane," Selecta Math. Sov. 1, 373-388.
Bogdanov, R. [1981b] "Versal deformations of a singular point on the plane in the case of zero eigenvalues," Selecta Math. Sov. 1, 389-421.

Davidowicz, P., Gliwicz, Z. M. \& Gulati, R. D. [1988] "Can Daphnia prevent a blue-green algal bloom in hypertrophic lakes? A laboratory test," Limnologica 19, 21-26.

Freedman, H. I. \& Wolkowicz, G. S. K. [1986] "Predatorprey systems with group defence: The paradox of enrichment revisited," Bull. Math. Biol. 48, 493-508.

Freedman, H. I. [1987] Deterministic Mathematical Models in Population Ecology (HIFR Consulting Ltd., Edmonton).

Freedman, H. I. \& Ruan, S. [1992] "Hopf bifurcation in three-species food chain models with group defense," Math. Biosci. 111, 73-87.

Holmes, J. C. \& Bethel, W. M. [1972] "Modification of intermediate host behavior by parasites," Zoolog. J. Linnean Soc. 51 (Suppl. 1), 123-149.

Kooij, R. E. \& Zegeling, A. [1996] "A predator-prey model with Ivlev's functional response," J. Math. Anal. Appl. 198, 473-489.

Kooij, R. E. \& Zegeling, A. [1997] "Qualitative properties of two-dimensional predator-prey systems," Nonlin. Anal. 29, 693-715.

Luckinbill, L. S. [1973] "Coexistence in laboratory populations of Paramecium Aurelia and its predator Didinium Nastum," Ecology 54, 1320-1327.

Mischaikow, K. \& Wolkowicz, G. S. K. [1990] "A predator-prey system involving group defense: A connection matrix approach," Nonlin. Anal. 14, 955-969.

Rosenzweig, M. L. [1971] "Paradox of enrichment: Destabilization of exploitation ecosystems in ecological time," Science 171, 385-387.

Rothe, F. \& Shafer, D. S. [1991] "Multiple bifurcation in a predator-prey system with nonmonotonic predator response," Proc. R. Soc. Edinburgh A120, 313-347.

Ruan, S. \& Xiao, D. [2001] "Global analysis in a predator-prey system with nonmonotonic functional response," SIAM J. Appl. Math. 61, 1445-1472.

Schaffer, W. M. \& Rosenzweig, M. L. [1978] "Hamage to the red queen I. Coevolution of predators and their victims," Theoret. Population Biol. 14, 135-157.

Sugie, J., Kohno, R. \& Miyazaki, R. [1997] "On a predator-prey system of Holling type," Proc. Amer. Math. Soc. 124, 2041-2050.

Sugie, J. [1998] "Two-parameter bifurcation in a predator-prey system of Ivlev type," J. Math. Anal. Appl. 217, 349-371.

Tener, J. S. [1965] Muskoxen (Queen's Printer, Ottawa). Wolkowicz, G. S. K. [1988] "Bifurcation analysis of a predator-prey system involving group defence," SIAM J. Appl. Math. 48, 592-606.

Xiao, D. \& Ruan, S. [1999] "Bogdanov-Takens bifurcations in predator-prey systems with constant rate harvesting," Fields Inst. Commun. 21, 493-506. 